\title{
Comparison of quantum mechanical and classical trajectory calculations of cross sections for ion-atom impact ionization of negative - and positive -ions for heavy ion fusion applications
}

\author{
Igor D. Kaganovich, Edward A. Startsev and Ronald C. Davidson \\ Plasma Physics Laboratory, Princeton University, Princeton, NJ 08543
}

(Dated: August 9, 2021)

\begin{abstract}
Stripping cross sections in nitrogen have been calculated using the classical trajectory approximation and the Born approximation of quantum mechanics for the outer shell electrons of $3.2 \mathrm{GeV}$ $\mathrm{I}^{-}$and $\mathrm{Cs}^{+}$ions. A large difference in cross section, up to a factor of six, calculated in quantum mechanics and classical mechanics, has been obtained. Because at such high velocities the Born approximation is well validated, the classical trajectory approach fails to correctly predict the stripping cross sections at high energies for electron orbitals with low ionization potential.
\end{abstract}




\section{INTRODUCTION}

Ion-atom ionizing collisions play an important role in many applications, such as heavy ion inertial fusion [1], collisional and radiative processes in the Earth's upper atmosphere 2], ion-beam lifetimes in accelerators [3], atomic spectroscopy [4] and ion stopping in matter 5], and are also of considerable academic interest in atomic physics [6].

To estimate the ionization and stripping rates of fast ions propagating through gas or plasma, the values of ion-atom ionization cross sections are necessary. In contrast to the electron [7] and proton [8, 9] ionization cross sections, where experimental data or theoretical calculations exist for practically any ion and atom, the knowledge of ionization cross sections by fast complex ions and atoms is far from complete [10]. While specific values of the cross sections for various pairs of projectile ions and target atoms have been measured at several energies [11, 12, 13], the scaling of cross sections with energy and target or projectile nucleus charge has not been experimentally mapped.

There are several theoretical approaches to cross section calculations. These include: classical calculations that make use of a classical trajectory and the atomic electron velocity distribution functions given by quantum mechanics [this approach is frequently referred to as the classical trajectory Monte Carlo (CTMC) approach]; quantum mechanical calculations based on the Born, eikonal or quasiclassical approximations, and so forth [10]. All approaches are computationally intensive, and the error and range of validity have to be assessed carefully before making any approximations or applying the results.

Classical trajectory calculations are simpler to perform in comparison with quantum mechanical calculations. Moreover, in some cases the CTMC calculations yield results close to the quantum mechanical calculations [11, 14, 15]. The reason for similar results lies in the fact that the Rutherford scattering cross section is identical in both classical and quantum mechanical derivations [16]. Therefore, when an ionizing collision is predominantly a consequence of electron scattering at small impact parameters close to the nucleus, the quantum mechanical uncertainty in the scattering angle is small compared with the angle itself, and the classical calculation can yield an accurate description [17, 18]. But this is not always a case, as we demonstrate below. For fast projectile velocities and low ionization potentials, the difference between the classical and quantum mechanical calculations of ionization cross section can be as large as a factor of six for parameters to relevant to heavy ion fusion cross sections.

In the present analysis, we consider at first only the stripping cross section of loosely bound electron orbitals of $\mathrm{I}^{-}$and $C s^{+}$ions colliding with a neutral atom of nitrogen, or with a fully stripped nitrogen ion with $Z_{T}=7$ (for comparison). Atomic units are used throughout this paper with $e=\hbar=m_{e}=1$, which corresponds to length normalized to $a_{0}=\hbar^{2} /\left(m_{e} e^{2}\right)=0.529 \cdot 10^{-8} \mathrm{~cm}$, velocity normalized to $v_{0}=e^{2} / \hbar=2.19 \cdot 10^{8} \mathrm{~cm} / \mathrm{s}$, and energy normalized to $E_{0}=m_{e} v_{0}^{2}=2 R y=27.2 \mathrm{eV}$, where $R y$ is the Rydberg energy. The normalizing coefficients are retained in all equations for robust application of the formulas. For efficient manipulation of the formulas, it is worth noting that the normalized velocity is $v / v_{0}=0.2 \sqrt{E[k e V / a m u]}$, where $E$ is energy per nucleon in $k e V / a m u$. Therefore, $25 \mathrm{keV} / \mathrm{amu}$ corresponds to the atomic velocity scale.

The typical scale for the electron orbital velocity with ionization potential $I_{n l}$ is $v_{n l}=$ $v_{0} \sqrt{2 I_{n l} / E_{0}}$. Here, $n, l$ is the standard notation for the main quantum number and the orbital angular momentum quantum number [16]. The collision dynamics is very different depending on whether $v$ is smaller or larger than $v_{n l}$. 


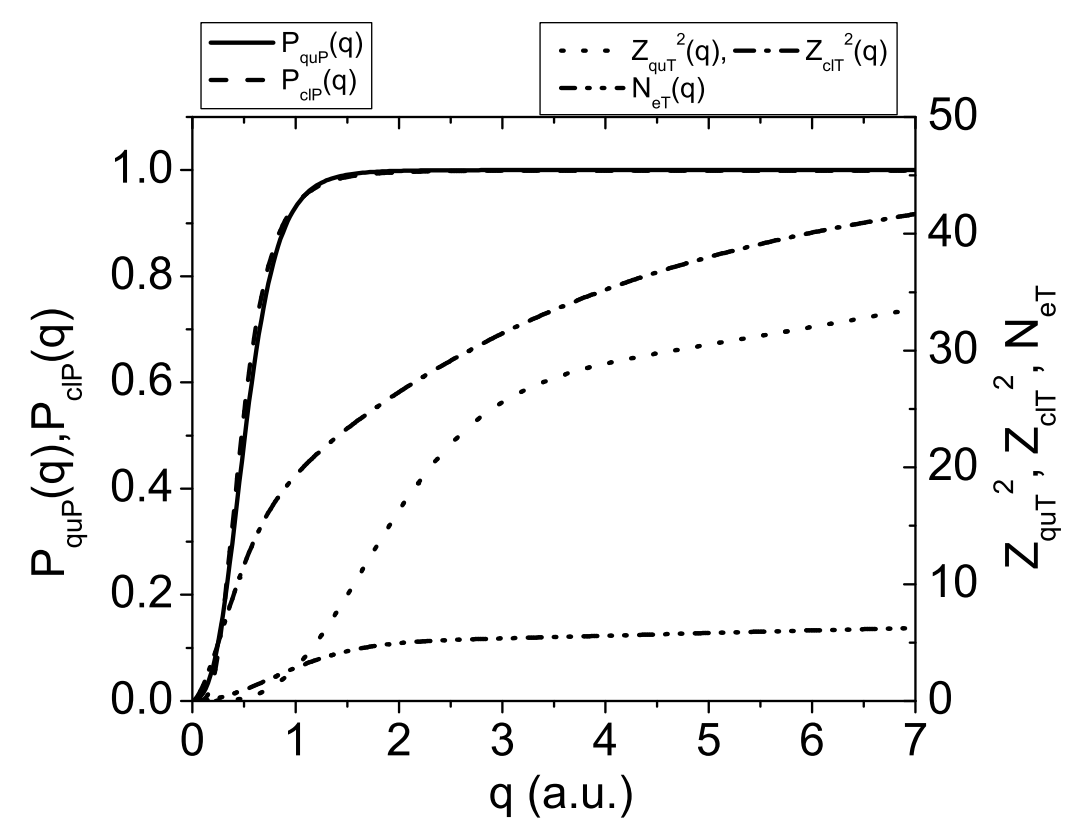

FIG. 1: Shown in the figure is a comparison of the ionization probabilities $\left[P_{q u P}(q)\right.$ in Eq.(2), and $P_{c l P}(q)$ in Eq.(3) $]$ and the effective charges $\left[Z_{q u T}(q) N_{e T}(q)\right.$ in Eq.(17), and $Z_{c l T}(q)$ in Eq.(10)] in quantum and classical mechanics for $3.2 \mathrm{GeV} I^{-}$ions colliding with a nitrogen atom. Ionization of only the outer electron shell is considered (here, $I_{n l P}=3 \mathrm{eV}$ ).

\section{BEHAVIOR OF CROSS SECTIONS AT LARGE VALUES OF PROJECTILE VELOCITY $v>v_{n l}$}

When $v>>v_{n l}$, the projectile interaction with the target atom occurs for a very short time, and the interaction time decreases as the velocity increases. For $3.2 \mathrm{GeV} \mathrm{I}^{-}$ions, envisioned for heavy ion fusion applications, the projectile velocity in atomic units is $32 v_{0}$, while the electron orbital velocity is $v_{n l}=0.5 v_{0}$ for the first $(3.06 \mathrm{eV})$ ionization potential of $I^{-}$, and $v_{n l}=1.3 v_{0}$ for the first $(22.4 \mathrm{eV})$ ionization potential of $\mathrm{Cs}^{+}$. Therefore, we shall use the limit $v>>v_{n l}$.

In the limit, where $v>v_{0} Z_{T}$ and $v>>v_{n l}$, the Born approximation of quantum mechanics can be used [14, 16]. The first inequality assures that the nitrogen atomic potential can be taken into account as a small perturbation (the Born approximation); the second inequality allows us to use the unperturbed atomic wave function.

In both classical mechanics and in the Born approximation, the ionization cross section can be recast in the form [10, 15, 19, 20],

$$
\sigma=\int_{0}^{\infty} P_{P}(q) \frac{d \sigma}{d q} d q
$$

where $P_{P}(q)$ is the probability of electron stripping from the projectile when the electron acquires the momentum $q$, and $d \sigma / d q$ is the differential cross section for scattering with momentum $q$. 


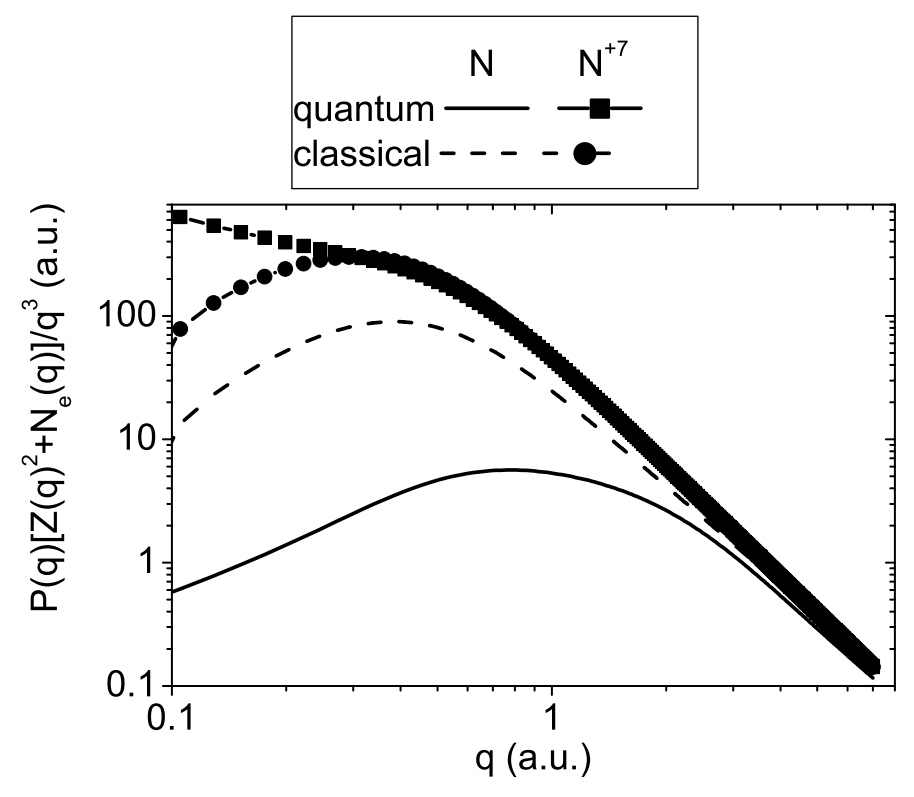

FIG. 2: Plots of differential cross sections for stripping of $I^{-}$ions by nitrogen atoms and fully stripped ions.

In quantum mechanics, $P_{q u P}(q)$ can be expressed by the square of the corresponding matrix element of transition from the initial state $\mid n l>$ to the state of the ejected electron $\mid \mathbf{k}>$ with momentum $\mathbf{k}$, integrated over all $\mathbf{k}$. This gives

$$
P_{q u P}(q)=\int|<n l| e^{i \mathbf{q} \cdot \mathbf{r}}|\mathbf{k}>|^{2} d^{3} \mathbf{k} .
$$

The analytical form of $P_{q u P}(q)$ for hydrogen-like electron functions is given in Ref. [19]. In classical mechanics, $P_{c l P}(q)$ is given by the integral over the electron velocity distribution function $f\left(\mathbf{v}_{e}\right)$ defined by

$$
P_{c l P}(q)=\int \Theta\left(\mathbf{q} \cdot \mathbf{v}_{e}+\frac{q^{2}}{2 m_{e}}-I_{n l}\right) f\left(\mathbf{v}_{e}\right) \mathbf{d} \mathbf{v}_{e} .
$$

Classical mechanics prescribes the electron velocity distribution function (EVDF) for hydrogen-like orbitals as a microcanonical ensemble, where

$$
f\left(\mathbf{v}_{e}\right)=C v_{e}^{2} \int \delta\left(\frac{m_{e} v_{e}^{2}}{2}-\frac{e^{2} Z_{T}}{r}+I_{n l}\right) r^{2} d r .
$$

Here, $C$ is a normalization constant defined so that $\int f\left(v_{e}\right) d v_{e}=1$, and $\delta(\ldots)$ denotes the Dirac delta-function. Interestingly, the EVDF for a hydrogen-like electron orbitals is identical in both the quantum mechanical and classical calculations [16], with

$$
f\left(v_{e}\right)=\frac{32 v_{n l}^{7}}{\pi} \frac{v_{e}^{2}}{\left[v_{e}^{2}+v_{n l}^{2}\right]^{4}}
$$


where $v_{n l}$ is the scale of the electron orbital velocity defined by

$$
v_{n l}=v_{0} \sqrt{2 I_{n l} / E_{0}}
$$

In the Born approximation of quantum mechanics, $d \sigma / d q$ is given by [16, 21]

$$
\frac{d \sigma}{d q}=8 \pi a_{0}^{2} \frac{v_{0}^{2}\left(m_{e} v_{0}\right)^{2}}{v^{2}} \frac{Z_{q u T}^{2}(q)+N_{e T}(q)}{q^{3}},
$$

where

$$
Z_{q u T}(q)=\left|Z_{T}-\sum_{n l} F_{n l T}(q)\right|, N_{e T}(q)=\left[N_{e T}^{\text {total }}-\sum_{n l T}\left|F_{n l T}(q)\right|^{2}\right] .
$$

Here, $Z_{q u T}(q)$ is the effective charge, subscript $q u$ stands for quantum mechanics, $F_{n l T}(q)=$ $\int e^{i \mathbf{q} \cdot \mathbf{r}} \rho_{n l T}(r) d^{3} \mathbf{r}$ is the form factor of the target atom's orbital $n l$ with the electron density $\rho_{n l T}(r)$, and $N_{e T}^{\text {total }}$ is the total number of electrons in the target atom $\left[N_{e T}(q \rightarrow \infty)=\right.$ $\left.N_{e T}^{\text {total }}\right]$.

In classical mechanics, $d \sigma / d q$ is given by

$$
\frac{d \sigma}{d q}=2 \pi \rho \frac{d \rho}{d q}
$$

Here, $\rho(q)$ is the impact parameter for a collision resulting in the momentum transfer $q$. For fast collisions, $q$ is mainly perpendicular to the projectile velocity, and $q$ is determined by integration of the electric field of the target atom on the electron, which gives

$$
q(\rho)=-\frac{2 \rho}{v} \int_{\rho}^{\infty} \frac{d U_{T}}{d r} \frac{1}{\sqrt{r^{2}-\rho^{2}}} d r
$$

where $U_{T}(r)$ is the atomic potential of the target atom. To compare the classical calculation with the quantum mechanical calculation, we recast Eqs. (8) and (9) into a form similar to Eq.(6), introducing the effective charge $Z_{c l T}(q)$ defined by

$$
Z_{c l T}(q)=\frac{q v}{2 m_{e} a_{0} v_{0}^{2}} \sqrt{-q \rho(q) \frac{d \rho}{d q}}
$$

where subscript $\mathrm{cl}$ stands for classical mechanics. Note that for the bare target ion, $U_{T}=$ $-e^{2} Z_{T} / r$ and $Z_{c l T}(q)=Z_{T}$. Finally, making use of the effective charge in Eq.(10), the differential cross section in classical mechanics takes on a form similar to Eq.(6) in quantum mechanics, i.e.,

$$
\frac{d \sigma}{d q}=8 \pi a_{0}^{2} \frac{v_{0}^{2}\left(m_{e} v_{0}\right)^{2}}{v^{2}} \frac{Z_{c l T}(q)^{2}+N_{e T}^{\text {total }}}{q^{3}} .
$$

Here, the final term accounts for ionization by the $N_{e T}^{t o t a l}$ target electrons.

Figure 1 shows a comparison of the ionization probabilities $\left[P_{q u P}(q)\right.$ in Eq.(2), and $P_{c l P}(q)$ in Eq.(3)] and the effective charges $\left[Z_{q u T}(q)\right.$ in Eq.(17), and $Z_{c l T}(q)$ in Eq.(10)] in quantum mechanics and classical mechanics for $3.2 \mathrm{GeV} I^{-}$ions colliding with a nitrogen atom. Ionization of only the outer electron shell is considered (here, $I_{n l P}=3.06 \mathrm{eV}$, approximating as a hydrogen-like orbital). 
Figure 2 shows that for stripping by neutral atoms, the main contributions arise from intermediate momenta in the range $q=0.5-1$, while for stripping by the bare target nucleus, small values of $q$ make the largest contribution to the cross section, which corresponds to large impact parameters (due to the Coulomb long-range interaction). Because $P_{q u P}>P_{c l P}$ for $q<<1$, but $Z_{q u T}<Z_{c l T}(q)$, the quantum mechanical cross sections are larger than the classical stripping cross sections for stripping by the bare nucleus, but smaller than the classical stripping cross sections for the atoms. Carrying out the integration in Eq. (11) gives the stripping cross sections for only one electron from the outer electron shell for different ions with the same velocity $v=32 v_{0}$ colliding with a nitrogen atom. The results are shown in Table 1 for $3.2 \mathrm{GeV} \mathrm{I}^{-}$ions; in Table 2 for $3.35 \mathrm{GeV} \mathrm{Cs}{ }^{+}$ions; and in Table 3 for $25 \mathrm{MeV} \mathrm{H}^{-}$.

$\begin{array}{lll}\sigma, 10^{-16} \mathrm{~cm}^{2} & \text { quantum } & \text { classical } \\ \mathrm{N} & 0.08 & 0.47 \\ \mathrm{~N}^{+7} & 2.5 & 1.29\end{array}$

Table 1. Cross section for stripping of $3.2 \mathrm{GeV} \mathrm{I}^{-}$ions colliding with a nitrogen atom and a fully stripped nitrogen ion ( stripping of only one electron from the outer electron shell is considered here with $\left.I_{n l P}=3.06 \mathrm{eV}\right)$.

$\begin{array}{lll}\sigma, 10^{-16} \mathrm{~cm}^{2} & \text { quantum } & \text { classical } \\ \mathrm{N} & 0.045 & 0.10 \\ \mathrm{~N}^{+7} & 0.32 & 0.17\end{array}$

Table 2. Cross section for stripping of $3.35 \mathrm{GeV} \mathrm{Cs}{ }^{+}$ions (the same velocity as $3.2 \mathrm{GeV}$ $I^{-}$) colliding with a nitrogen atom or a fully stripped nitrogen ion ( stripping of only one electron from the outer electron shell is considered here with $I_{n l P}=22.4 \mathrm{eV}$ ).

$\begin{array}{lll}\sigma, 10^{-16} \mathrm{~cm}^{2} & \text { quantum } & \text { classical } \\ \mathrm{N} & 0.10 & 1.34 \\ \mathrm{~N}^{+7} & 12.5 & 5.05\end{array}$

Table 3. Cross section for stripping of $25 \mathrm{MeV} \mathrm{H}^{-}$ions (the same velocity as $3.2 \mathrm{GeV}$ $I^{-}$) colliding with a nitrogen atom or a fully stripped nitrogen ion ( stripping of only one electron from the outer electron shell is considered here with $I_{n l P}=0.75 \mathrm{eV}$ ).

Figure 3 shows the same results as in Fig.2, but the results are obtained for $3.35 \mathrm{GeV} \mathrm{Cs}{ }^{+}$ ions (ionization of only one outer electron shell is considered here with $I_{n l P}=22.4 \mathrm{eV}$ ). Note that $3.35 \mathrm{GeV} \mathrm{Cs}{ }^{+}$is chosen to have the same velocity as a $3.2 \mathrm{GeV} \mathrm{I}^{-}$ion.

In the limit $v>>v_{n l}$, the stripping cross section by a fully stripped ion can be analytically evaluated. The Bohr formula, derived by means of classical mechanics, neglects the electron atomic velocity, and gives for the cross section [17]

$$
\sigma^{\text {Bohr }}\left(v, I_{n l}, Z_{p}\right)=2 \pi Z_{p}^{2} a_{0}^{2} \frac{v_{0}^{2} E_{0}}{v^{2} I_{n l}}
$$

Accounting for the electron atomic velocity gives an additional factor of 5/3 [15]. The Bethe formula [19] derived by means of the Born approximation of quantum mechanics gives

$$
\sigma^{\text {Bethe }}=\sigma^{\text {Bohr }}\left(v, I_{n l}, Z_{p}\right)\left[0.566 \ln \left(\frac{v}{v_{n l}}\right)+1.261\right] .
$$




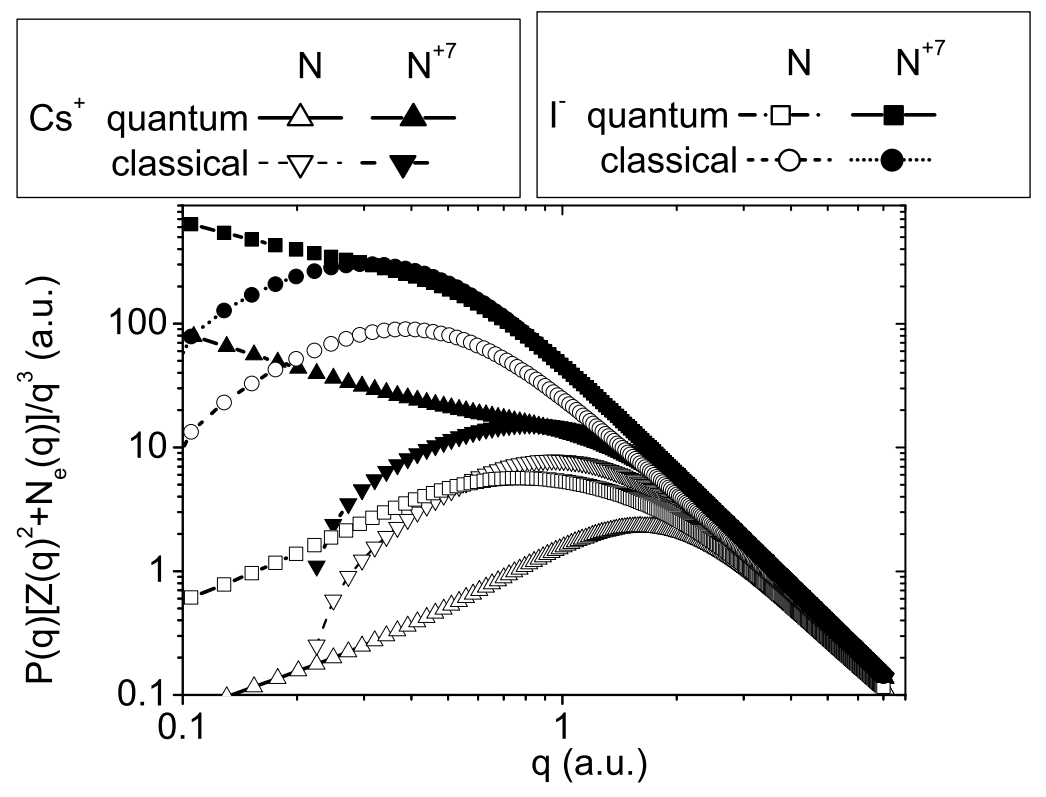

FIG. 3: Plots of the differential cross sections of ionization for $\mathrm{Cs}^{+}$and $\mathrm{I}^{-}$ions by nitrogen atoms and fully stripped ions.

The results of cross sections calculations using Eq.(12) with a factor $5 / 3$ and the result in Eq.(13) coincide with the results in Tables 1, 2 and 3 of stripping cross sections by a fully stripped nitrogen ions calculated in classical trajectory approximation and the Born approximation of quantum mechanics, respectively.

The stripping cross sections calculated in classical trajectory approximation for $\mathrm{Cs}^{+}$and $I^{-}$ions by fully stripped nitrogen ions is only factor 2-3 larger than the stripping cross sections by neutral nitrogen atoms, which is in qualitative agreement with the observations in Ref.[12]. However, there is a large difference, up to a factor 30, in the stripping cross sections calculated in the Born approximation of quantum mechanics.

It is evident that the stripping of $\mathrm{Cs}^{+}$ions by fully stripped nitrogen ions decreases by a factor of $22.4 \mathrm{eV} / 3 \mathrm{eV}=7.5$ compared with $I^{-}$ions, which is in agreement with the Bohr [Eq.(12)] and Bethe [Eq.(13)] formulas. The stripping cross sections for $\mathrm{Cs}^{+}$and $\mathrm{I}^{-}$ions by neutral nitrogen atoms differ by only a factor of 2 . In classical mechanics, because the interaction potential is a strong function of the separation, to transfer a considerably larger momentum requires a rather small decrease in impact parameter. This is why, notwithstanding the large difference in ionization potential by a factor of 7 , the difference between the two cross sections is only a factor of 2 . Table 3 shows that the difference between the quantum and classical treatments increases for smaller ionization potentials (compare Table 3 with Table 1).

The reason for such a large difference between the quantum mechanical and classical mechanical stripping cross sections for $I^{-}$can be easily understood from the example of elastic electron scattering from the shielded Coulomb potential $U(r)=\exp \left(-r / a_{0}\right) / r$. The differential cross section for elastic scattering is shown in Fig.4 . For the shielded Coulomb 




FIG. 4: Plots of the differential cross sections for the shielded Coulomb potential for $v=32 v_{0}$.

potential, direct application of the Born approximation gives [16]

$$
\frac{d \sigma}{q d q}=8 \pi a_{0}^{2} \frac{v_{0}^{2}\left(m_{e} v_{0}\right)^{2}}{v^{2}} \frac{1}{\left(q^{2}+m_{e}^{2} \hbar^{2} / a_{0}^{2}\right)^{2}},
$$

and the total cross section is $\sigma=4 \pi a_{0}^{2} v_{0}^{2} / v^{2}$. The total classical cross section, obtained from integrating $\int \rho d \rho$, diverges because of the contributions from large $\rho$ (small $q$ ). Evidently, the quantum mechanical cross section departs from the Rutherford scattering formula for $q /\left(m_{e} v_{0}\right)<1$, whereas the classical mechanical cross section departs from the Rutherford scattering formula only for $q /\left(m_{e} v_{0}\right)<2 v_{0} / v$ [see Eq.(91) and Fig.4]. Therefore, the classical differential cross section differs from the quantum mechanical result by a factor of $\left[v /\left(2 v_{0}\right)\right]^{4}$, which for $v=32 v_{0}$ gives a difference in small-angle differential cross section of up to a factor of $10^{4}$ (see Fig.4).

Tables 4 and 5 are similar to Tables 1 and 2, but the calculations are carried out for ion energies 30 times smaller, in the range of $100 \mathrm{MeV}$. Table 5 shows that the predictions of the classical and quantum mechanical theories are similar for $100 \mathrm{MeV}$ ions. However, they are a factor two different for $I^{-}$ions, and the cross sections are the same within $10 \%$ accuracy for $\mathrm{Cs}^{+}$ions. The contribution from small $q$ to the stripping cross section by a neutral nitrogen atom is smaller for $\mathrm{Cs}^{+}$ions than for $\mathrm{I}^{-}$ions, thereby significantly reducing the stripping cross section of $\mathrm{Cs}^{+}$ions compared with $\mathrm{I}^{-}$ions, especially for the calculation in the classical trajectory approximation (see Tables 4 and 5, and Fig.5).

$$
\begin{array}{lll}
\sigma, 10^{-16} \mathrm{~cm}^{2} & \text { quantum } & \text { classical } \\
\mathrm{N} & 2.47 & 6.8 \\
\mathrm{~N}^{+7} & 61 & 37
\end{array}
$$

Table 4. Cross section for the stripping of $105 \mathrm{MeV} I^{-}$ions $\left(v=5.75 v_{0}\right)$ colliding with a nitrogen atom and a fully stripped nitrogen ion (stripping of only one electron from the 


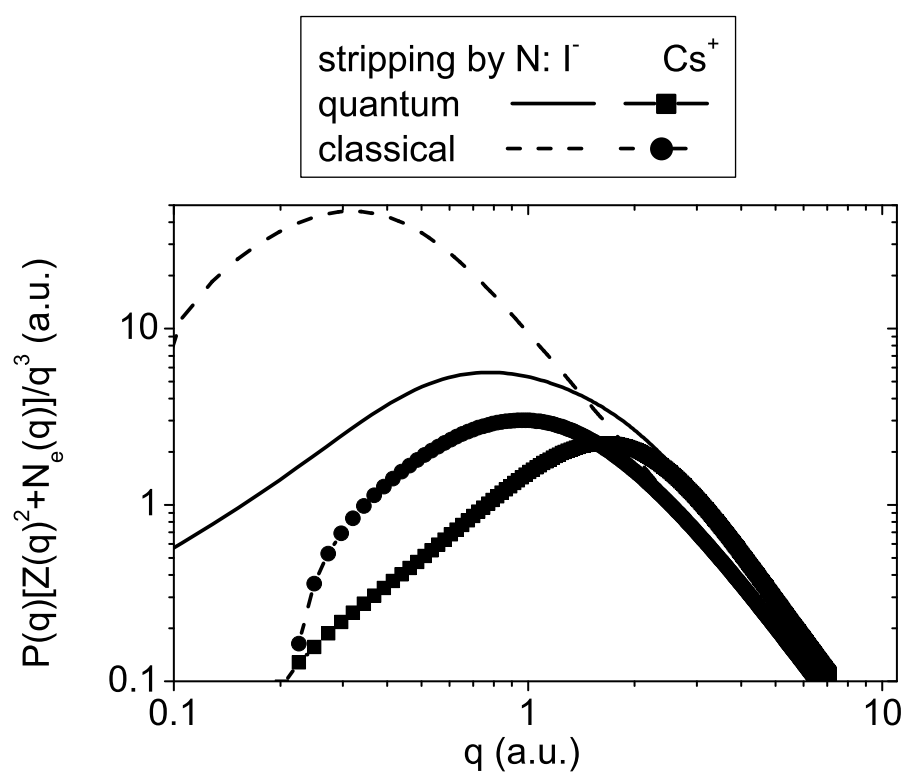

FIG. 5: Plots of the differential cross sections for stripping of $100 \mathrm{MeVCs} s^{+}$and $105 \mathrm{MeV} I^{-}$ions $\left(v=7.5 v_{0}\right)$ by nitrogen atoms.

outer electron shell is considered here with $I_{n l P}=3 \mathrm{eV}$ ).

$\begin{array}{lll}\sigma, 10^{-16} \mathrm{~cm}^{2} & \text { quantum } & \text { classical } \\ \mathrm{N} & 1.36 & 1.4 \\ \mathrm{~N}^{+7} & 6.6 & 5.2\end{array}$

Table 5. Cross section for the stripping of $110 \mathrm{MeV} \mathrm{Cs} s^{+}$ions $\left(v=5.75 v_{0}\right)$ colliding with a nitrogen atom and a fully stripped nitrogen ion (stripping of only one electron from the outer electron shell is considered here with $\left.I_{n l P}=22.4 \mathrm{eV}\right)$.

\section{CALCULATION OF TOTAL CROSS SECTIONS}

The total stripping cross section is defined as

$$
\sigma^{\text {total }}=\sum_{m} m \sigma_{m}
$$

where $\sigma_{m}$ is the cross section for stripping $m$ electrons in each collision. This cross section is convenient to use for electron production calculations. The stripping cross section for any degree of ionization is defined as

$$
\sigma=\sum_{m} \sigma_{m}
$$

which is a convenient expression to use to determine the ion confinement time in an accelerator. In the limit $v>>v_{n l}$, the calculation of the total stripping cross section can be performed assuming that the stripping from different electron orbitals occurs independently 
[10], i.e.,

$$
\sigma^{t o t a l}=\sum_{n l} N_{n l} \sigma_{n l}
$$

where $\sigma_{n l}$ is the stripping cross section of only one electron from the electron orbital $n l$, and $N_{n l}$ is the number of electrons in the orbital. The structure of the electron orbitals for $I^{-}$ions is shown in Table 6.

$\begin{array}{lllllllllll}n l & 5 \mathrm{p} & 5 \mathrm{~s} & 4 \mathrm{~d} & 4 \mathrm{p} & 4 \mathrm{~s} & 3 \mathrm{~d} & 3 \mathrm{p} & 3 \mathrm{~s} & 2 \mathrm{p} & 2 \mathrm{~s} \\ \mathrm{~N}_{n l} & 6 & 2 & 10 & 6 & 2 & 10 & 6 & 2 & 6 & 2 \\ \mathrm{I}_{n l} & 3.08 & 13.2 & 50.1 & 125.0 & 185.83 & 623.26 & 892.5 & 1.07 \mathrm{e} 3 & 4.65 \mathrm{e} 3 & 5.2 \mathrm{e} 3 \\ \sigma_{n l}\left(v=32 v_{0}\right) & 0.080 & 0.054 & 0.030 & 0.018 & 0.013 & 5.5 \mathrm{e}-3 & 4.2 \mathrm{e}-3 & 3.6 \mathrm{e}-3 & 8.3 \mathrm{e}-4 & 7.3 \mathrm{e}-4 \\ \sigma_{n l}\left(v=5.75 v_{0}\right) & 2.45 & 1.65 & 0.92 & 0.52 & 0.39 & 0.12 & 0.078 & 0.062 & 5.8 \mathrm{e}-3 & 4.6 \mathrm{e}-3\end{array}$

Table 6. The structure of electron orbitals for $I^{-}$ions and the individual cross sections avaluated for an orbital electron in units of $10^{-16} \mathrm{~cm}^{2}$.

Here, $n l$ denotes the atomic orbital quantum numbers, $\mathrm{I}_{n l}$ is the ionization potential in $\mathrm{eV}$, and $\sigma_{n l}$ denotes the individual cross section for an orbital electron in units of $10^{-16} \mathrm{~cm}^{2}$. The sum over all orbitals gives $\sigma^{\text {total }}=1.1 \cdot 10^{-16} \mathrm{~cm}^{2}$ for $3.2 \mathrm{GeV} I^{-}$ions. To correctly account for multiple ionization, the inclusion of multi-electron effects is necessary. This will be addressed in a future publication. However, it is clear that the stripping cross section for any degree of ionization by neutral atoms is limited by the geometrical cross section of the atom (the geometrical cross section of a nitrogen atom is much smaller than the geometrical cross section of a $\mathrm{Cs}^{+}$ion or a $I^{-}$ion 22$]$ ). The nitrogen atom geometric cross section is $\sigma_{N}=1.5 \cdot 10^{-16} \mathrm{~cm}^{2}[22]$, and therefore $\sigma<\sigma_{N}$ is expected. Preliminary estimates suggest that single electron stripping is expected under these conditions.

For $105 \mathrm{MeV} I^{-}$ions, however, the sum over all orbitals gives $\sigma^{\text {total }}=33 \cdot 10^{-16} \mathrm{~cm}^{2}$, whereas $\sigma_{N}=1.5 \cdot 10^{-16} \mathrm{~cm}^{2}$. This indicates that multi-electron ionization is expected. However, it is clear that the stripping cross section for any degree of ionization is limited from above by $\sigma_{N}=1.5 \cdot 10^{-16} \mathrm{~cm}^{2}$.

The structure of the electron orbitals for $\mathrm{Cs}^{+}$ions, and the individual cross sections for an orbital electron in units of $10^{-16} \mathrm{~cm}^{2}$ are illustrated in Table 7 . Note that a $\mathrm{Cs}^{+}$ion has the same number of electrons on each orbital as a $I^{-}$ion.

$\begin{array}{lllllllllll}n l & 5 \mathrm{p} & 5 \mathrm{~s} & 4 \mathrm{~d} & 4 \mathrm{p} & 4 \mathrm{~s} & 3 \mathrm{~d} & 3 \mathrm{p} & 3 \mathrm{~s} & 2 \mathrm{p} & 2 \mathrm{~s} \\ \mathrm{~N}_{n l} & 6 & 2 & 10 & 6 & 2 & 10 & 6 & 2 & 6 & 2 \\ \mathrm{I}_{n l} & 22.4 & 34.0 & 88.3 & 176 & 242 & 742 & 1.03 \mathrm{e} 3 & 1.2 \mathrm{e} 3 & 5.1 \mathrm{e} 3 & 5.7 \mathrm{e} 3 \\ \sigma_{n l}\left(v=32 v_{0}\right) & 0.044 & 0.037 & 0.022 & 0.014 & 0.011 & 4.8 \mathrm{e}-3 & 3.7 \mathrm{e}-3 & 3.2 \mathrm{e}-3 & 7.4 \mathrm{e}-4 & 6.5 \mathrm{e}-4 \\ \sigma_{n l}\left(v=5.75 v_{0}\right) & 1.35 & 1.12 & 0.66 & 0.41 & 0.32 & 0.098 & 0.065 & 0.052 & 4.7 \mathrm{e}-3 & 3.8 \mathrm{e}-3\end{array}$

Table 7. The structure of electron orbitals for $\mathrm{Cs}^{+}$ions and the individual cross sections for an orbital electron in units of $10^{-16} \mathrm{~cm}^{2}$.

For $3.35 \mathrm{GeV} \mathrm{Cs}^{+}$ions colliding with a nitrogen atom with velocity $v=32 v_{0}$ $(25 \mathrm{MeV} / \mathrm{amu})$, the summation in Eq. (17) over all orbitals gives $\sigma^{\text {total }}=0.72 \cdot 10^{-16} \mathrm{~cm}^{2}$. This estimate of the cross section is consistent with Olson's result in Ref.[12], $\sigma=2 \cdot 10^{-16} \mathrm{~cm}^{2}$ for $25 \mathrm{MeV} / \mathrm{amu} \mathrm{Xe} e^{+}$. Note that the factor of three difference between the results presented in Table 7 and the results in Ref. [12] is due to the fact that the cross sections in Table 7 
are predicted by making use of quantum mechanics, whereas results in Ref. [12] are classical trajectory calculations, not applicable at such high projectile velocities.

For $110 \mathrm{MeV} \mathrm{Cs}{ }^{+}$ions colliding with a nitrogen atom, $v=5.75 v_{0}(0.8 \mathrm{Mev} / \mathrm{amu})$ and the summation over all orbitals in Eq.(17) gives $\sigma^{\text {total }}=21 \cdot 10^{-16} \mathrm{~cm}^{2}$, whereas the geometrical cross section of a nitrogen atom is only $\sigma_{N}=1.5 \cdot 10^{-16} \mathrm{~cm}^{2}<<\sigma^{\text {total }}$. This indicates that multi-electron ionization is expected, similar to $I^{-}$ions at the same velocity. As noted earlier, to correctly account for multiple ionization, multi-electron calculations are necessary. However, it is clear that the stripping cross section $\sigma$ for any degree ionization is limited by $\sigma_{N}=1.5 \cdot 10^{-16} \mathrm{~cm}^{2}$. This estimate of the cross section is consistent with Olson's result 12], $\sigma^{\text {total }}=4 \cdot 10^{-16} \mathrm{~cm}^{2}$ for $2 \mathrm{MeV} /$ amu $X e^{+}$. The inequality $\sigma^{\text {total }}>\sigma_{N}$ indicates the important effect of multi-electron events.

\section{CONCLUSIONS}

For low ionization potential, where a small momentum transfer $q$ contributes to stripping, the classical approach is not valid. For $3.2 \mathrm{GeV} \mathrm{I}^{-}$ions, the classical trajectory approach overestimates by a factor of six the stripping cross section by atomic nitrogen, and by a factor of two the stripping cross section of $3.35 \mathrm{GeV} \mathrm{Cs}{ }^{+}$ions. For $110 \mathrm{MeV} \mathrm{Cs}{ }^{+}$ions and $105 \mathrm{MeV} I^{-}$ions colliding with a nitrogen atom at velocity $v=5.75 v_{0}(0.8 \mathrm{Mev} / \mathrm{amu})$, multi-electron ionization is expected. For a correct description of multiple ionization, multielectron calculations are necessary. However, it is clear that the stripping cross section for any degree of ionization is limited from above by the geometrical cross section of nitrogen, with $\sigma_{N}=1.5 \cdot 10^{-16} \mathrm{~cm}^{2}$, and should be be similar in magnitude for $I^{-}$ions and $\mathrm{Cs}^{+}$ions at energies in the $100 \mathrm{MeV}$ range. (The geometrical cross section of a nitrogen atom is much smaller than the geometrical cross section of a $C s^{+}$ion or a $I^{-}$ion [22]. This effect is similar to the hole produced by a bullet piercing a paper target, where the hole size is determined by the bullet cross section, not by the paper target.)

\section{Acknowledgments}

This research was supported by the U.S. Department of Energy. It is a pleasure to acknowledge the benefits of useful discussion with Christine Celata, Larry Grisham, Grant Logan and Art Molvik.

[1] B. G. Logan, C. M. Celata, J. W. Kwan, E. P. Lee, M. Leitner, P. A. Seidl, S. S. Yu, J. J. Barnard, A. Friedman, W. R. Meier, R. C. Davidson, Laser and Particle Beams 20, 369 (2002).

[2] G. M. Keating, S. W. Bougher, J. Geophys. Res.- Space Phys. 97 (A4), 4189 (1992).

[3] H. Beyer, V. P. Shevelko (eds), Atomic physics with Heavy Ions (Springer, Berlin 1999).

[4] A. Bogaerts, R. Gijbels, R. J. Carman, Spectrochimica Acta Part B - Atomic Spectroscopy 53, 1679 (1998).

[5] C. Stockl, O. Boine-Frankenheim, M. Geissel, M. Roth, H. Wetzler, W. Seelig, O. Iwase, P. Spiller, R. Bock, W. Suss, D. H. H. Hoffmann, Nucl. Instrum. Meth. A 415, 558 (1998).

[6] S. Datz, G. W. F. Drake, T. F. Galagher, H. Kleinpoppen, G. Zu Putlitz, Rev. Mod. Phys. 71, S223 (1999).

[7] G. S. Voronov, Atomic Data and Nuclear Data Tables 65, 1, (1997). 
[8] M. E. Rudd, Y. -K. Kim, D. H. Madison, and J. W. Galallagher, Rev. Mod. Phys. 64, 441 (1992).

[9] M. E. Rudd, Y. -K. Kim, D. H. Madison, and T. J. Gay, Rev. Mod. Phys. 57, 965 (1985).

[10] R. K. Janev, L. P. Presnyakov, V. P. Shevelko, Physics of Highly Charged Ions (Springer, Berlin 1999).

[11] D. Mueller, L. Grisham, I. Kaganovich, R. L. Watson, V. Horvat and K. E. Zaharakis, Physics of Plasmas, 8, 1753 (2001).

[12] R. E. Olson, R. L. Watson, V. Horvat, and K. E. Zaharakis, J. Phys. B: At. Mol. Opt. Phys. 35, 1893 (2002).

[13] R. L. Watson, Y. Peng, V. Horvat, G. J. Kim, and R.E. Olson, Phys. Rev.A 67, 022706 (2003).

[14] D. Mueller, L. Grisham, I. Kaganovich, R. L. Watson, V. Horvat, K. E. Zaharakis and Y. Peng, Laser and Particle Beams 20, 551 (2002).

[15] I. D. Kaganovich, E. Startsev and R. C. Davidson, "Ionization Cross-Sections in Ion-Atom Collisions for High Energy Ion Beams", Proceedings of the 2003 Particle Accelerator Conference, in preparation (2003).

[16] L. D. Landau and E. M. Lifshitz, Quantum Mechanics (Addison-Wesley Publishing Co., 1958).

[17] N. Bohr, K. Dan. Vidensk. Selsk. Mat.- Fys. Medd. 18, N8 (1948).

[18] I. D. Kaganovich, E. Startsev and R. C. Davidson, "Evaluation of Ionization Cross Sections in Energetic Ion-Atom Collisions," Proceedings of the 2001 Particle Accelerator Conference, (2001). http://accelconf.web.cern.ch/AccelConf/p01/PAPERS/TPAH314.PDF

[19] H. Bethe, Ann. Phys. (Leipz.) 5, 325 (1930).

[20] H. A. Bethe and R. Jackiw, Intermidiate Quantum Mechanics (The Benjamin/Cummings Publishing Company, sec. ed., 1968).

[21] V. P. Shevelko, I. Yu. Tolstikhina and Th. Stoehlker, Nucl. Instr. Meth. B 184, 295 (2001).

[22] Periodic Table of the Elements in CRC Handbook of Chemistry and Physics, 81st edition, 200-2001. 